\title{
Matthew Effect of the Random Drift on the Evolution of Cooperation
}

\author{
Chao Liu' and Rong $\mathrm{Li}^{2}$ \\ ${ }^{1}$ Department of Applied Mathematics, Shanghai University of Finance and Economics, Shanghai 200433, China \\ ${ }^{2}$ Department of Applied Mathematics, Shanghai Finance University, Shanghai 201209, China \\ Correspondence should be addressed to Chao Liu; liu.chao@shufe.edu.cn
}

Received 22 November 2013; Accepted 7 January 2014; Published 16 February 2014

Academic Editor: Wenwu Yu

Copyright (c) 2014 C. Liu and R. Li. This is an open access article distributed under the Creative Commons Attribution License, which permits unrestricted use, distribution, and reproduction in any medium, provided the original work is properly cited.

\begin{abstract}
The effect of the random drift on the evolutionary prisoner's dilemma game is studied on regular lattices. A new evolutionary rule is proposed, which stochastically combines the deterministic rule with the random drift rule. It is found that the random drift has an effect on the evolutionary dynamics depending on the values of the temptation-to-defect $b$ and the probability $p$ of the random drift. When the random drift occurs with low probabilities, which interests us more, a phenomenon of the Matthew effect on the evolution of cooperation is found. Explanations of this phenomenon are deduced through the analysis on the dynamics and pattern formations of the PDG system.
\end{abstract}

\section{Introduction}

Since the introduction of evolutionary game theory by Smith and Price $[1,2]$, one of the important issues is to understand the emergence and evolution of cooperation [3-6]. Among the many games, the most studied example is the prisoner's dilemma game (PDG), as it provides a simple example of the difficulties of cooperation [7-10]. The standard PDG is described by the following set of rules. When two players play a PDG, each of them can choose to cooperate (C) or defect (D). Each player will gain a payoff depending jointly on his choice and the opponent's choice. A cooperator receives $R$ when playing with a cooperator and $S$ when playing with a defector while a defector earns $P$ when playing with a defector, and $T$ against a cooperator, with $T>R>P>S$. Given this payoff ordering, in a well-mixed (unstructured) population where each agent interacts with all other agents (or a representative sample of the population composition), defectors are fitter and thus the fraction of cooperators asymptotically vanishes.

However, cooperation is widespread in many biological, social, and economic systems [11, 12]. One of the proposed mechanisms to explain this phenomenon is network reciprocity $[13,14]$. For example, early pioneering work on the
PDG in two-dimensional square lattices made the observation that, unlike in unstructured populations, cooperators and defectors can coexist in the lattice indefinitely $[15,16]$. Simply said, the clustering of cooperators in the lattice could provide high enough fitness to the cooperator nodes exposed to invasion, to the extent of preserving cooperators from evolutionary extinction, even when defection is favoured by the one-shot (two-players) game analysis [17]. A recent and powerful approach to studying such questions is provided by evolutionary graph theory [18].

All the game models incorporate some kind of evolutionary dynamics, which also play a crucial role in the results. As for $[15,16]$, the well-known promotion of cooperation enforced by spatial lattices is linked to a particular best-takesover update rule, in which each individual node plays with its immediate neighbours each time step accumulating a payoff; then it updates its strategy by imitating the one of the highest payoff in its neighbourhood, including itself.

The best-takes-over update rule is a nonstochastic imitation strategy. However, for real dynamical systems, external disturbances or system errors are generally inevitable. Therefore, it is worth considering the dependence of the promotion of cooperation on the evolutionary rules and its robustness against perturbations. Indeed, previous work has pursued this 


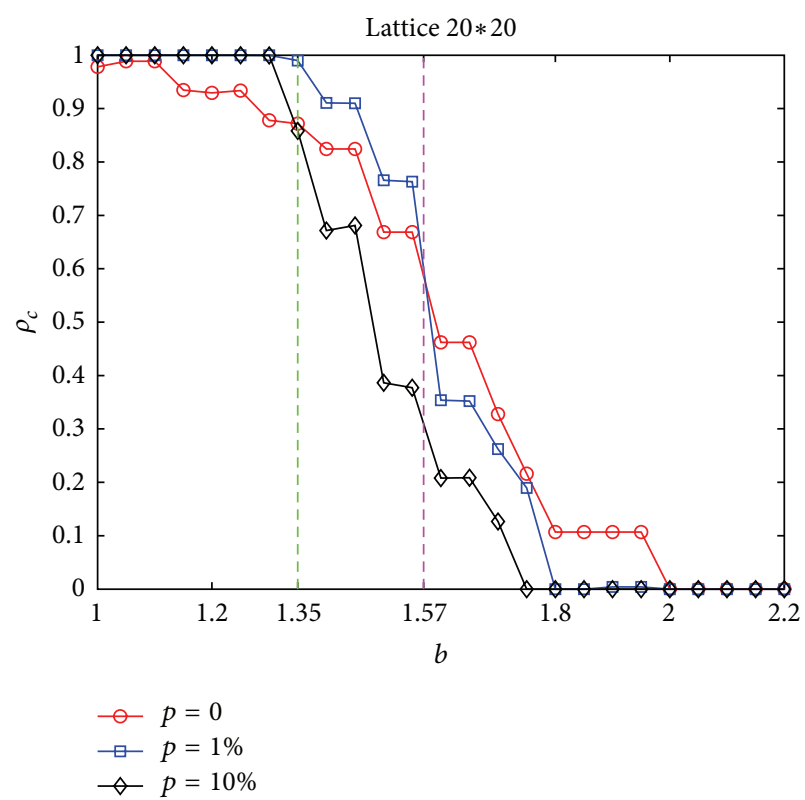

(a)

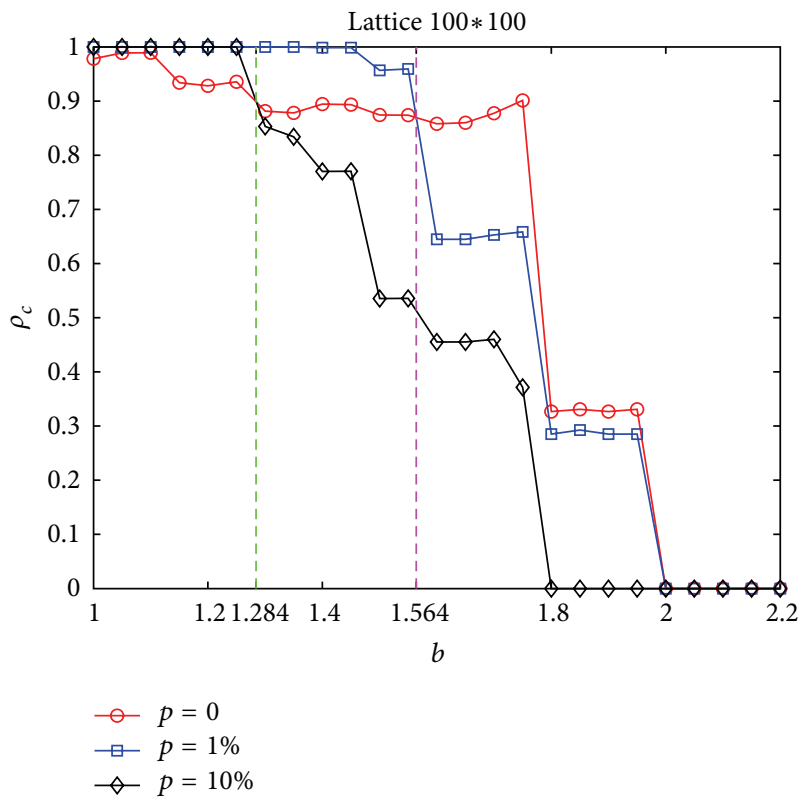

(b)

Figure 1: The frequency of cooperators, $\rho_{c}$, as a function of the temptation-to-defect $b$ in an evolutionary PDG on both $20 * 20$ and $100 * 100$ regular 8-neighbored lattices with periodic boundary conditions.

enquiry [19-21], replacing the deterministic update strategy with some stochastic imitative rules, finding greatly reduced cooperation levels.

In these game models, players are viewed as rational, who update their strategy by copying, within certain constraints, the strategy of those others that are doing better, or in game theoretical terms that are obtaining higher payoffs from the game. Then, what difference will be there in the evolution of cooperation if players occasionally behave irrationally, updating strategy with no concern of their payoffs. In this

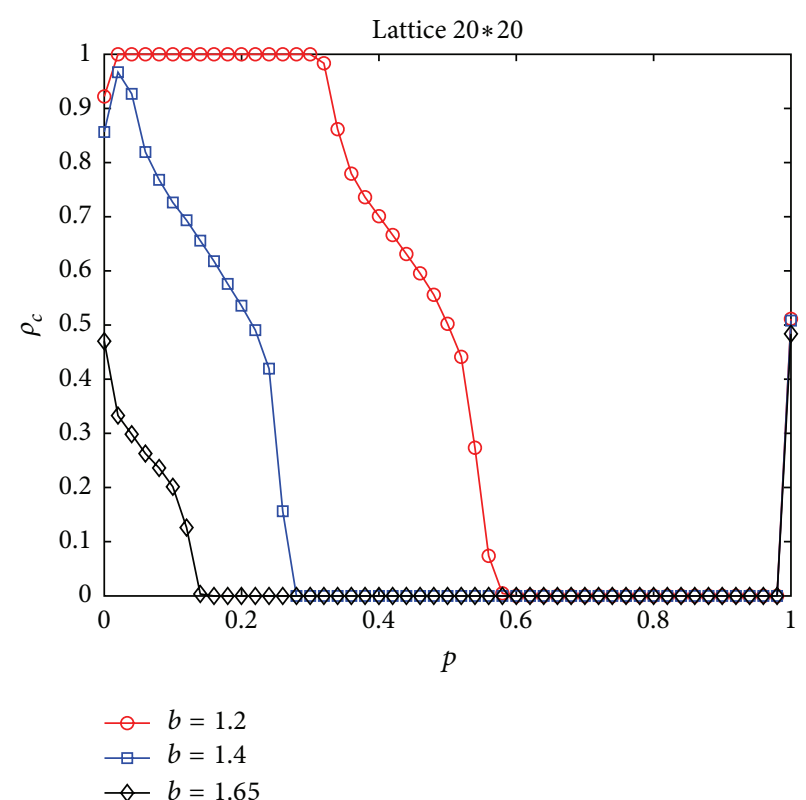

(a)

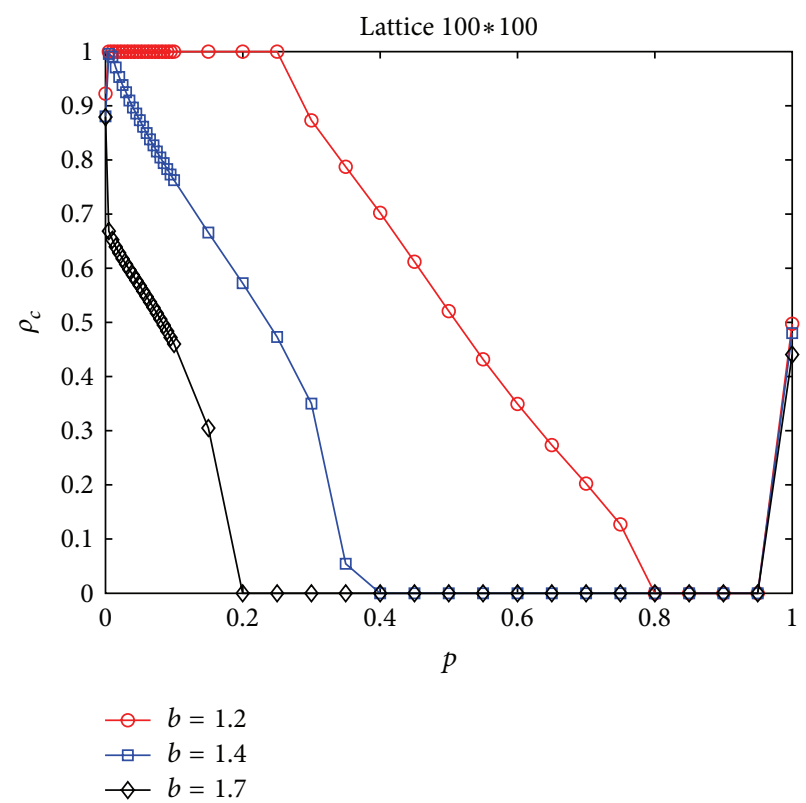

(b)

FIGURE 2: The frequency of cooperators as a function of the probability $p$ in an evolutionary PDG on $20 * 20$ and $100 * 100$ regular 8 -neighbored lattices with periodic boundary conditions.

paper, we will explore this problem by combining the besttakes-over rule with the random drift reproduction rule in the prisoner's dilemma game.

\section{The Model}

We study the PDG with pure strategies: the players can either defect (D) or cooperate (C). The same as in $[15,16]$, players are disposed on two-dimensional square lattices, and each player interacts only with its nearest neighbors and collects profits 


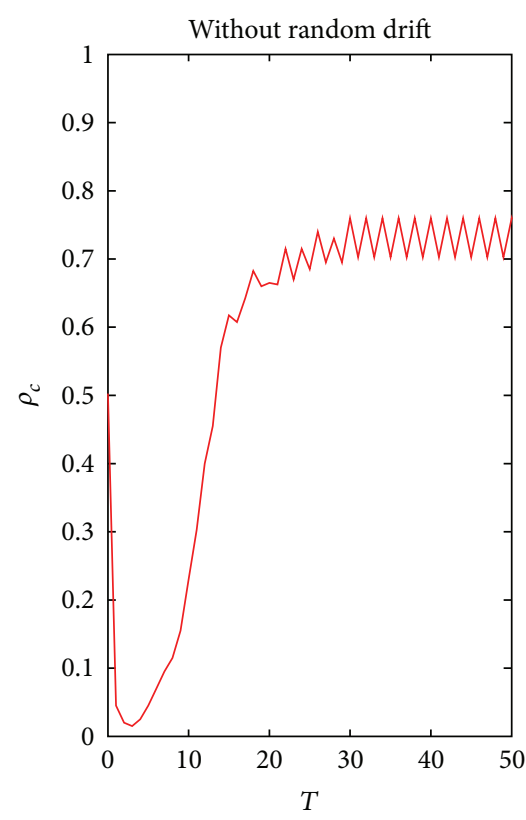

(a)

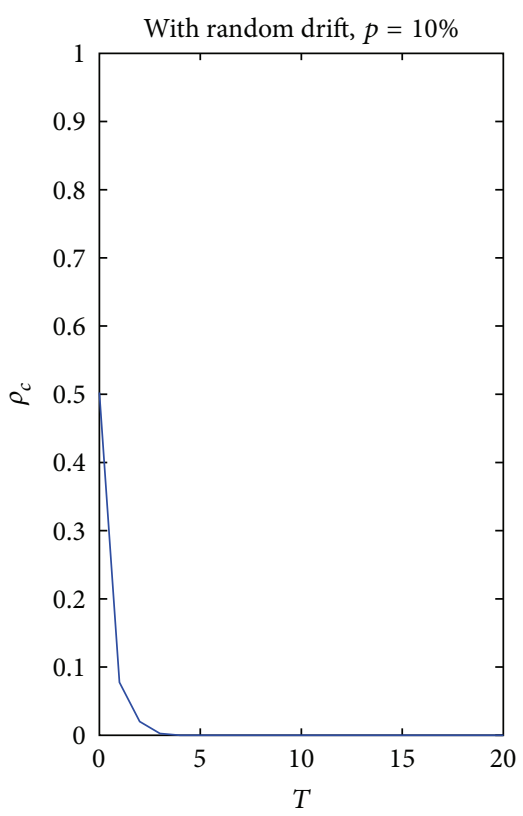

(b)

FIGURE 3: The dynamics of the PDG system with or without the influence of the random drift on a $20 * 20$ regular lattice. The temptation-todefect is $b=1.65$. All the simulations start from the same random initial configuration.

depending on the payoff parameters. Following common studies, the PDG is rescaled such that it depends on a single parameter; that is, the parameters are chosen to be $R=1$, $P=S=0$, and $T=b$, representing the advantage of defectors over cooperators (or the temptation-to-defect). After each round, the players are allowed to inspect their neighbors' payoffs and, according to the comparison, determine their strategies to adopt in the next round. Two alternative update rules determining the transformation of each player's strategy are described below.

(I) Best-takes-over: in each generation, an individual node imitates the strategy of one of its neighbors (including the node itself) that received the highest payoff in the last round. The best-takes-over rule is a deterministic rule according to which the individual with the highest gain in a given neighborhood reproduces with certainty.

(II) Random drift: whenever an individual node $i$ is updated, one neighbor $j$ is drawn at random among all its neighbors (including node $i$ itself), and node $i$ imitates the strategy of node $j$ with certainty regardless of the payoff of node $i$ or $j$ in the last round. Thus, for the random drift update rule, reproduction ability or fitness is not correlated with payoff but is correlated with the proportion of one strategy in the neighborhood.

To investigate how the random drift affects evolutionary games, we propose a new evolutionary rule which stochastically combines the deterministic rule with the random drift rule. A parameter of probability $p \in[0,1]$ is used in the choosing of the two alternative rules. That is, in each generation, an individual node updates its strategy by the best-takesover rule with probability $1-p$ and by the random drift rule with probability $p$.

Our simulations are carried out on the regular 8-neighbored square lattices. Initially, the cooperative and defective strategies are randomly distributed among the players with equal probability $1 / 2$. A synchronous updating scheme is adopted. Equilibrium frequencies of cooperators are obtained by averaging over 1000 generations after a transient time of 50000 generations. Each data point results from an average over 100 realizations of the initial conditions.

\section{Results and Discussion}

Figure 1 shows the varying frequency of cooperation versus temptation-to-defect $b$ on $20 * 20$ and $100 * 100$ regular lattice, respectively. $\rho_{c}$ represents the density of cooperators. The random drift update rule is employed with different probabilities $p$, where $p=0$ means the pure deterministic imitative dynamics. As shown in Figure 1, the random drift has obvious influence on the evolution of cooperation. Moreover, the influence depends on the value of the parameter $b$.

Despite the huge gap of the two lattices in size, the simulation results are qualitatively similar. In the following, we mainly analyze the results on the $20 * 20$ lattice as an example. Based on the different influence of the random drift, the range of parameter $b$ can be roughly divided into three regions. The first region is the small value region $b \in(1,1.35)$. In this parameter region, the phenomenon of stochastic resonance is observed. For small temptation-to-defect $b$, the frequency 


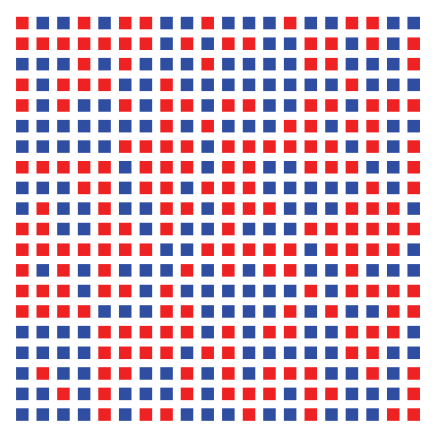

(a)

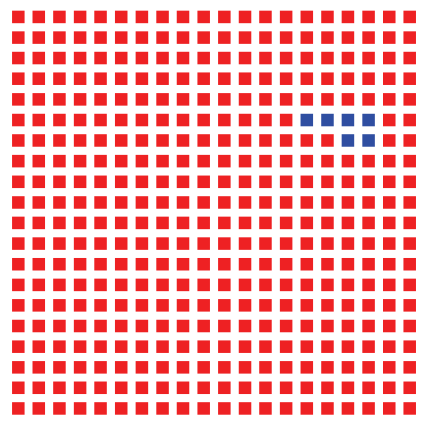

(d)

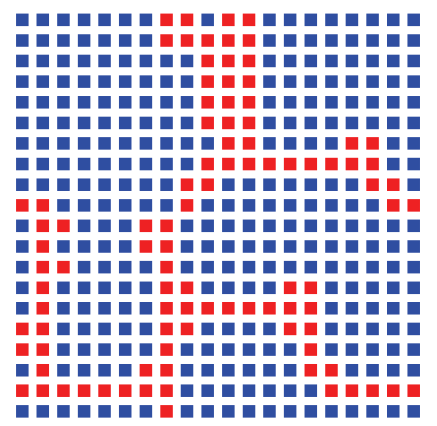

(g)

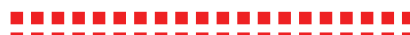

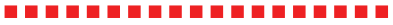

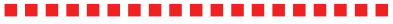

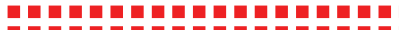

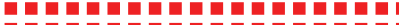

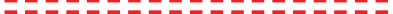
-

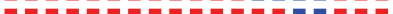

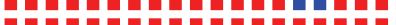

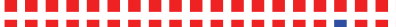

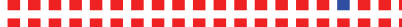

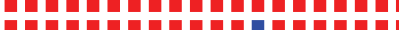

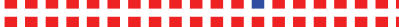

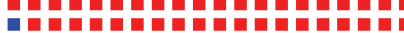

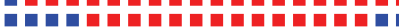

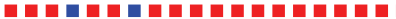
ต

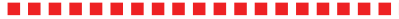
믐ำ

(b)

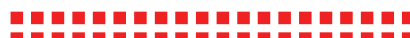

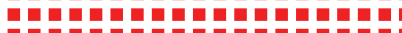

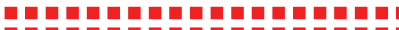

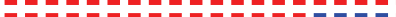
ต

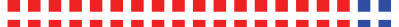

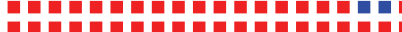
ต

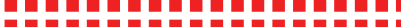

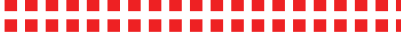

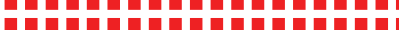
ตำ

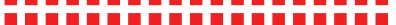

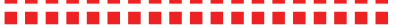

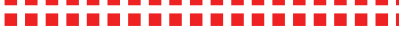

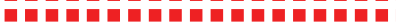

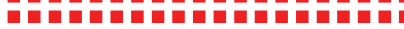

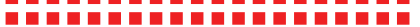

(e)

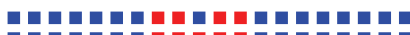

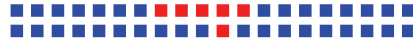

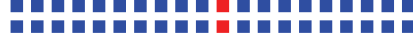

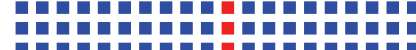

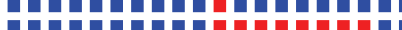

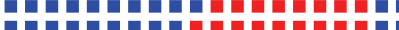

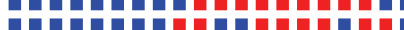

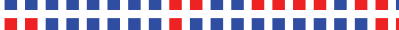

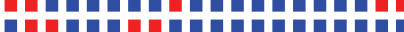

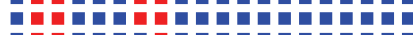

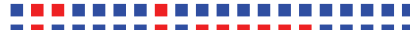

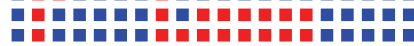

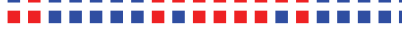

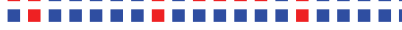

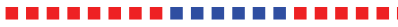

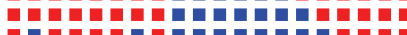

(h)

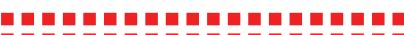

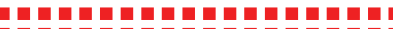

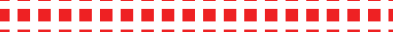

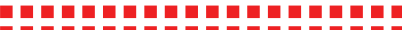

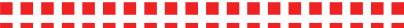

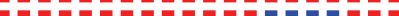

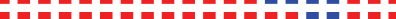

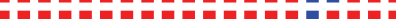

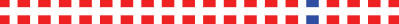

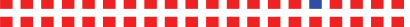

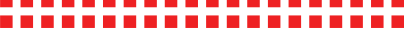

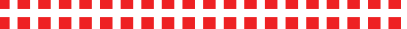

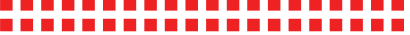

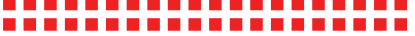

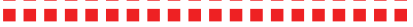

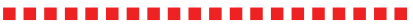

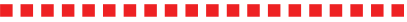

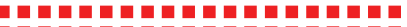

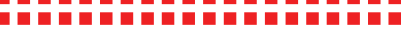

(c)

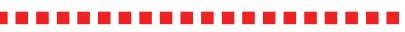

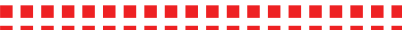

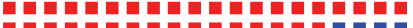

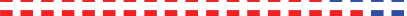

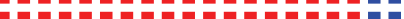

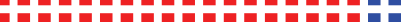

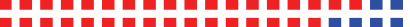

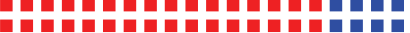

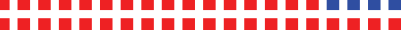

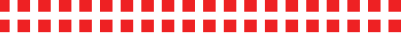

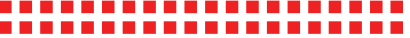

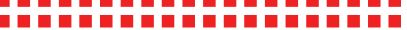

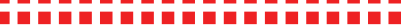

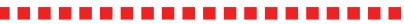

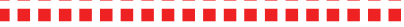

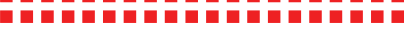

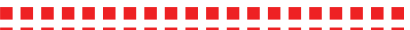

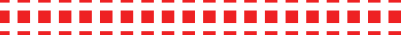

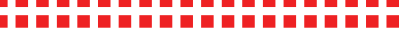

(f)

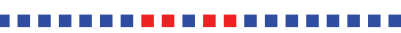

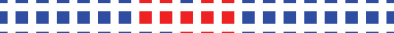

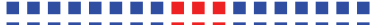

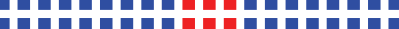

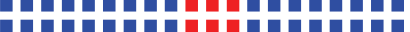

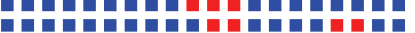

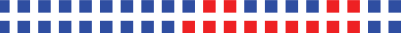

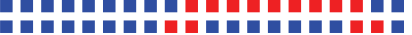

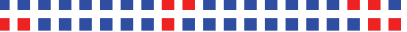

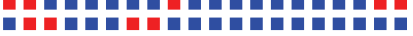

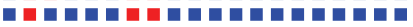

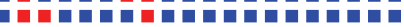

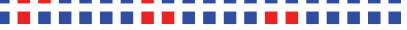

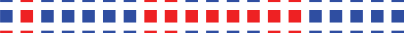

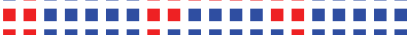

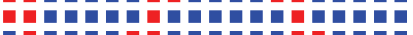

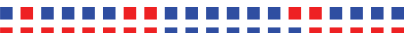

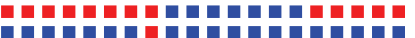

(i)

FIgURE 4: The pattern formation of the PDG system without the influence of the random drift on a $20 * 20$ regular lattice. The temptationto-defect is $b=1.65$. Color code: cooperators blue, defectors red.

of cooperation is high under the pure best-takes-over mechanism. However, it is still raised when the random drift is applied with small probabilities, even to an all-C state. The second parameter region is the moderate value region $b \in$ $(1.35,1.57)$. In this region, whether the random drift favours cooperation depends on the value of the probability $p$. When the random drift update rule is applied with probability $p=$ $1 \%$, the density of cooperators is higher than the one with the pure deterministic rule. However, when the probability rises to $p=10 \%$, the frequency of cooperation decreases lower than the deterministic one. $b \in(1.57,2.2)$ is the large parameter region. In this region, the evolutionary survival of cooperators is hindered by the random drift update rule. In particular, the parameter region $b \in(1.8,2)$ is worth of more attention. It is the chaotic parameter region under the deterministic mechanism, where the cooperators and defectors can coexist indefinitely; see [16] for details. As the random drift is applied, the chaotic evolution of the dynamic system is broken down, leading to an all-D state.
To have a more clear knowledge of the influence of the random drift on the evolution of cooperation, the variation of $\rho_{c}$ as a function of the probability $p$ is given in Figure 2 . Because of the qualitatively similarity, we still focus on the results of the $20 * 20$ lattice. The three values of the temptation-to-defect $b$ are from the three parameter regions mentioned above. As shown in Figure 2, for all the three values of $b$, the random drift is not favourable to the maintenance of cooperation when the probability $p$ is large enough. In particular, an all-D state prevails to form a flat valley in each of the three curves. When $p$ reaches $100 \%$, the equilibrium frequency of cooperation fluctuates around the original frequency (50\%). It is because, in this situation, the PDG evolves entirely under the random drift mechanism, for which payoffs of nodes do not count and the chance of survival for cooperators and defectors are equal. In the case $b=1.65$, the frequency of cooperation decreases monotonously with the increasing probability $p$ of the random drift. In the case $b=$ 1.2 or $b=1.4$, where $b$ is relatively small, an earlier raised and 


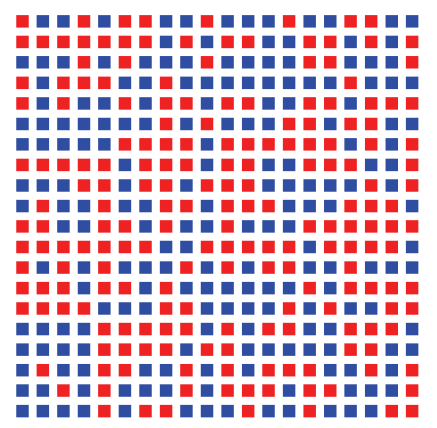

(a)

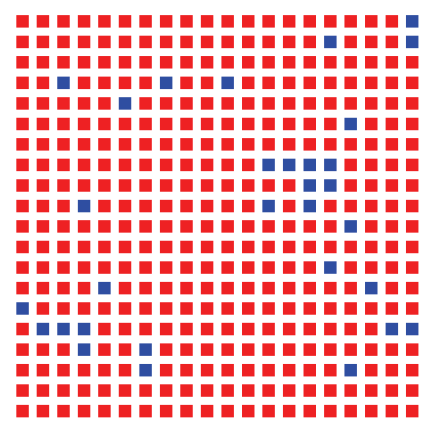

(b)

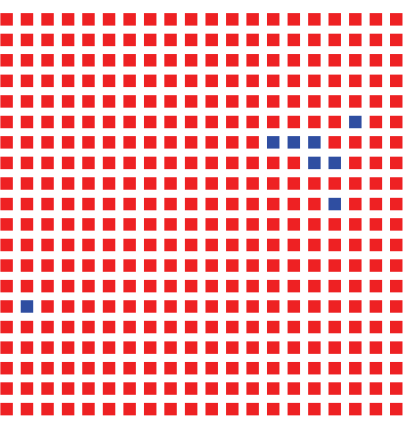

(c)

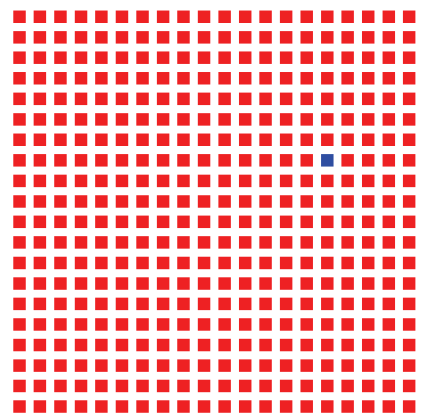

(d)

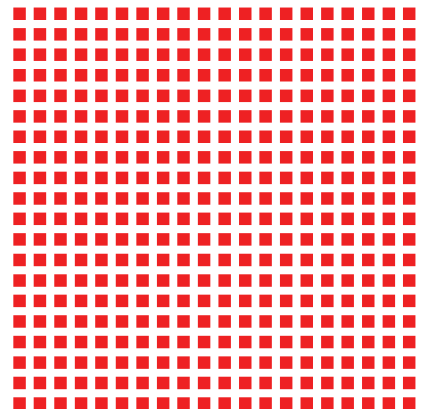

(e)

FIGURE 5: The pattern formation of the PDG system with the influence of the random drift on a $20 * 20$ regular lattice. $b=1.65$ and $p=10 \%$. Color code: cooperators blue, defectors red.

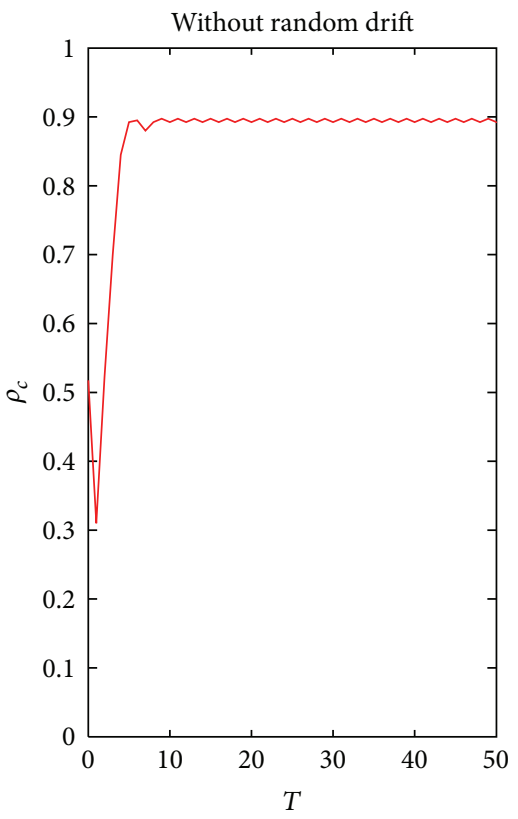

(a)

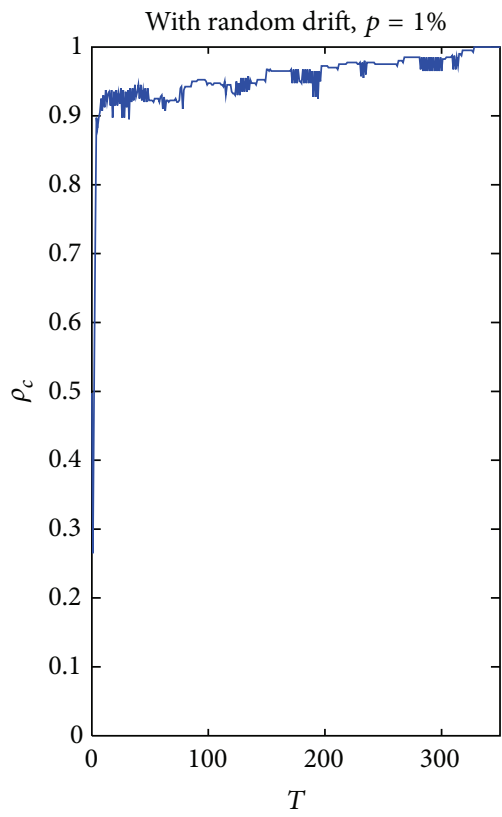

(b)

FIgURE 6: The dynamics of the PDG system with or without the influence of the random drift on a $20 * 20$ regular lattice. The temptation-todefect is $b=1.2$. All the simulations start from the same random initial configuration.

later decreased frequency of cooperation is found. Similar results are found on the $100 * 100$ lattice.

Thus, in fact, the random drift has an influence on the evolutionary PDG depending on the values of the temptation-to-defect $b$ and the probability $p$. When $p$ is high, which means that the random drift occurs frequently, it tends to depress cooperation for all values of $b$. When the random drift occurs only with low probabilities, which interests us more, a conclusion can be drawn as follows: when the temptation for players to defect is relatively small, with cooperators prevailing in the original PDG, the applied random drift tends to further enhance cooperation; under the opposite 


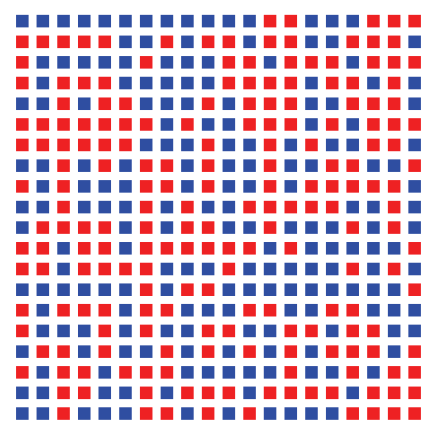

(a)

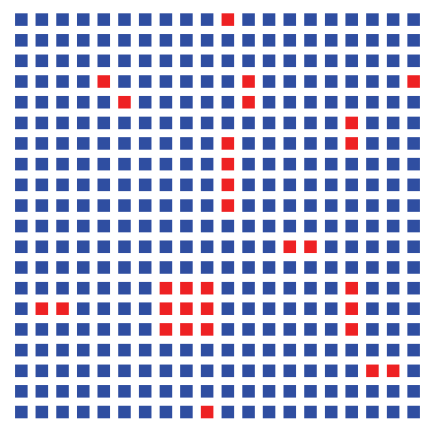

(d)

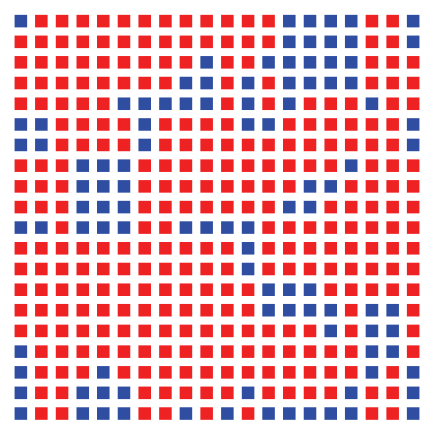

(b)

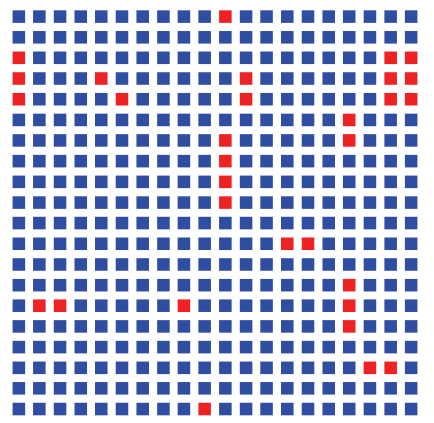

(e)

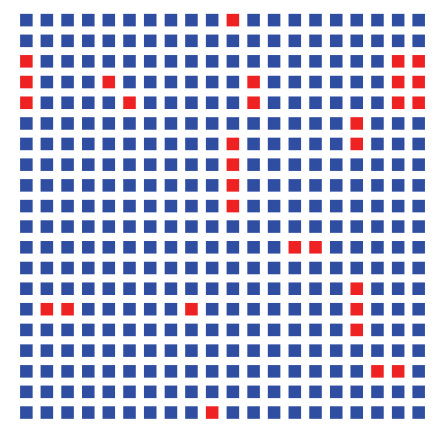

(c)

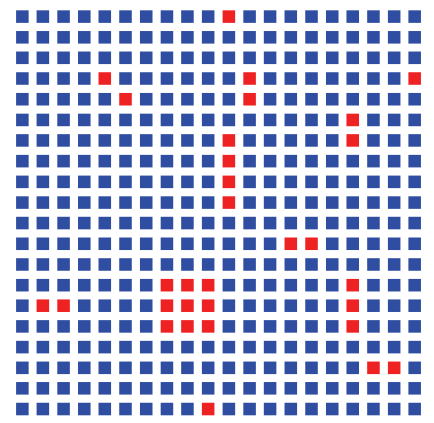

(f)

Figure 7: The pattern formation of the PDG system without the influence of the random drift on a $20 * 20$ regular lattice. The temptationto-defect is $b=1.2$. Color code: cooperators blue, defectors red.

conditions which is cooperation unfavourable, the random drift tends to further depress cooperation. In other words, for the cases with small $p$, the effect of the random drift on the evolution of cooperation is the so-called Matthew effect, which makes the rich richer.

How come this kind of Matthew effect arises when the random drift is applied to the deterministic imitative mechanism with small probability? The dynamics and the pattern formation of the PDG system may shed light on the explanation.

Setting $b=1.65$ and starting from the same initial distribution of cooperators, Figure 3 displays the dynamics of the PDG system with and without the influence of the random drift on the $20 * 20$ lattice. Under the pure deterministic imitation mechanism, the system settles at a period 2 oscillator after a short-term fluctuation as shown in Figure 3(a). In Figure 3(b), when the random drift update rule is applied with a probability $p=10 \%$, the cooperation is obviously suppressed and the system soon converges to an all-D state.

The corresponding pattern formations of the above two evolutionary processes are displayed in Figures 4 and 5, respectively. Figure 4(a) shows the initial pattern formation of the PDG system, in which cooperators and defectors randomly distributed in the lattice with equal probability. At the second time generation, the density of cooperators drops dramatically due to the high temptation to defectors. After that, the frequency of cooperators grows up gradually for the reason that the square shaped $\mathrm{C}$-clusters can survive and even grow in this region of parameter $b$, as displayed in Figures 4(b)-4(f). At last, the system regularly switches between two different pattern formations as shown in Figures 4(g)4(i), corresponding to the period 2 oscillator in Figure 3(a). As for Figure 5, although starting from the same initial configuration, cooperators vanish soon. From Figure 5(b) to Figure $5(\mathrm{c})$, one node (node $(7,17)$ ) of a $2 \times 2$ square shaped Ccluster turns to a defector due to the effect of the random drift, which leads to the breakdown of the entire square shaped Ccluster and eventually to the extermination of all cooperators.

The dynamics of the PDG system with temptation-todefect $b=1.2$ is displayed in Figure 6. Under the deterministic imitative mechanism, the dynamical system quickly settles at a period 2 oscillator with a high average equilibrium frequency of cooperators. When the random drift update rule is employed with a probability $p=1 \%$, the phenomenon of intermittent period oscillations appears in the dynamics, where the period oscillators become unstable due to the influence of the randomness. Finally, the system reaches an all-C state.

Figures 7 and 8 illustrate the dynamics of Figure 6 with pattern formations. As shown in Figures 7(c)-7(f), for the small value of $b$, defectors can only survive and remain stable in straight or oblique lines. At the same time, as shown at the bottom-left parts of Figures 7(c)-7(f), a single defector $(1 D)$ will grow to form a $3 \times 3$ square shaped $D$-cluster $(9 D)$ and then return to a single defector $(1 D)$ in the next time generation, which explains the period 2 oscillation of the dynamical system in Figure 7. However, when the random drift is applied with probability $p=1 \%$, both the stable lines 


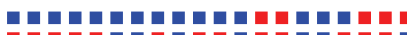

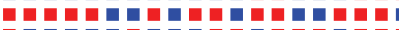

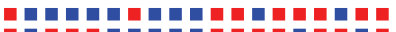

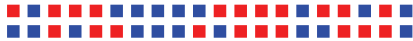

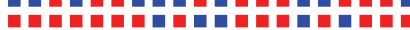
ตำ

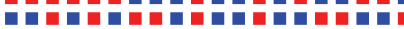

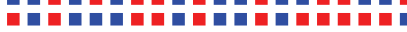

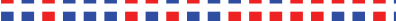
-

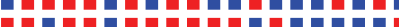
ตำ

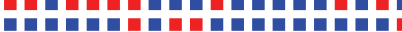

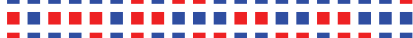

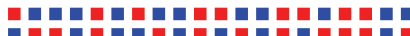

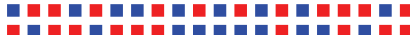

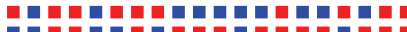

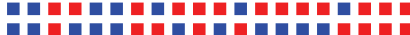
(a)

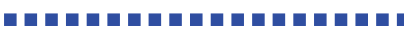

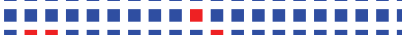

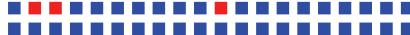

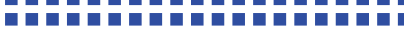

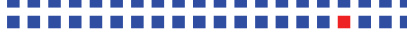

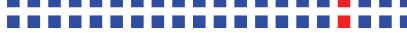

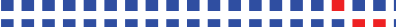

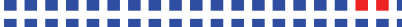

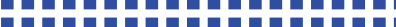

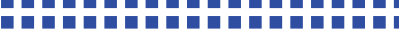

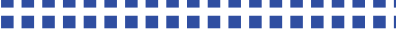

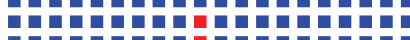

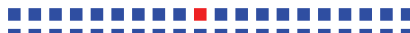

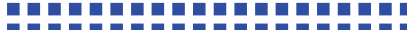

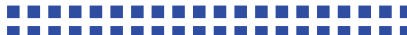

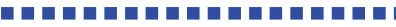

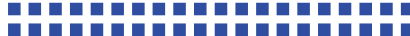

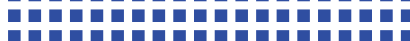
(d)

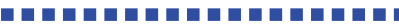
ตำ

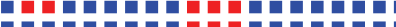
ตำ ตัต

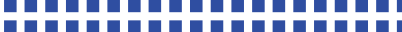

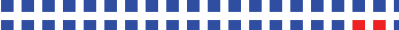

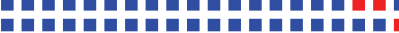

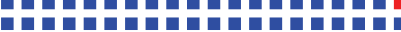

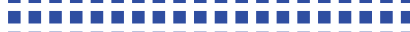

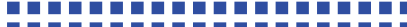

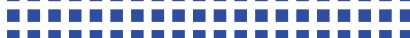

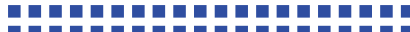

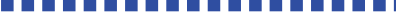

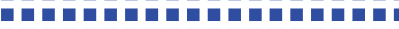

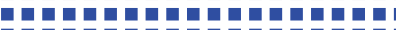

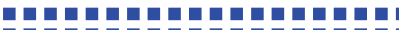

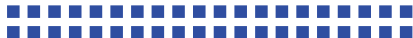
(g)

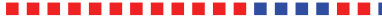
c)

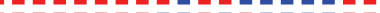
ต

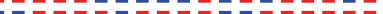
-

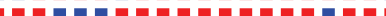

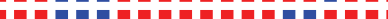

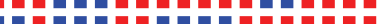
10

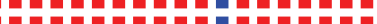

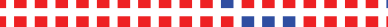
10ำ

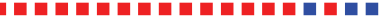
ต

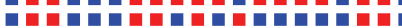

(b)

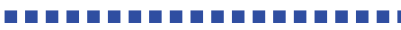

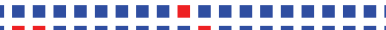
ต

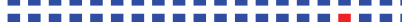
ำ-

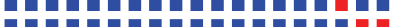
ตำ

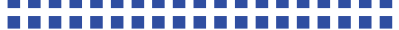

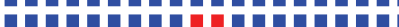

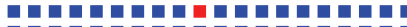

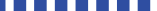

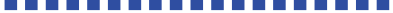

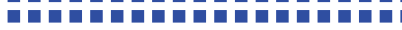
ต

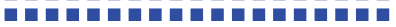

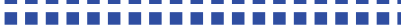

(e)

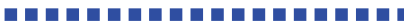

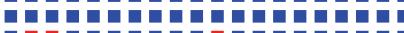

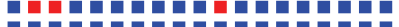

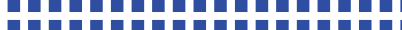
ตำang ต

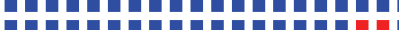
ง

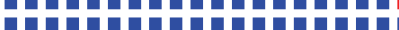

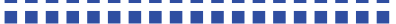

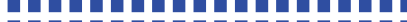

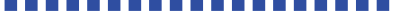

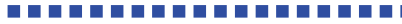

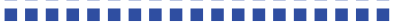

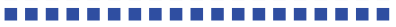

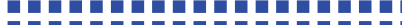

(h)

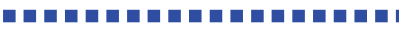

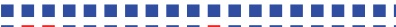

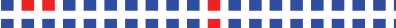
ตำ

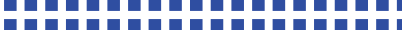

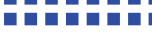

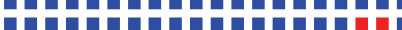

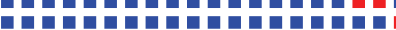

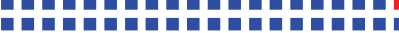

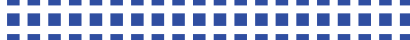

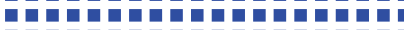

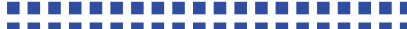

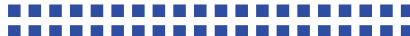

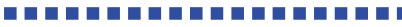

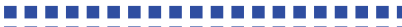

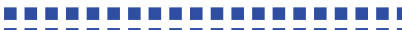

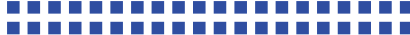

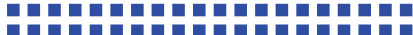

(j)

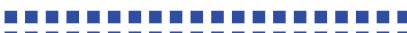

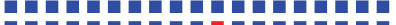

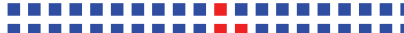

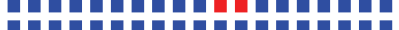

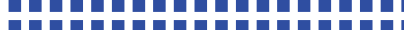

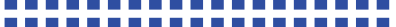

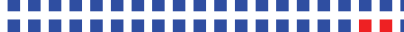

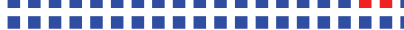

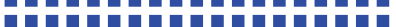

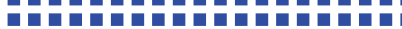

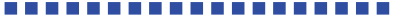

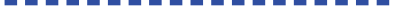

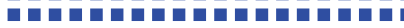

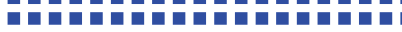

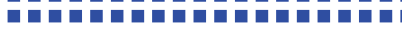

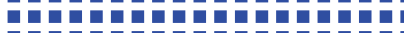

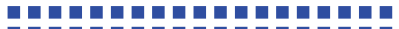

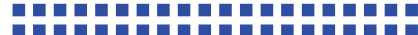

(k)

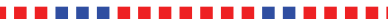

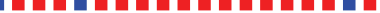
ตำ

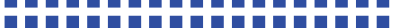

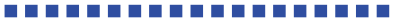

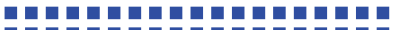

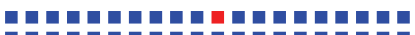

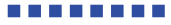

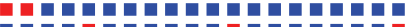
ตัต

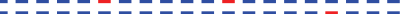

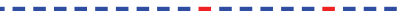

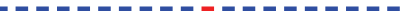

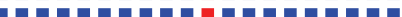

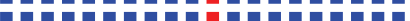

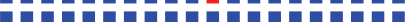

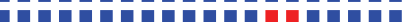

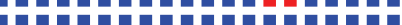

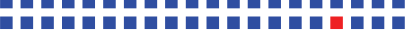

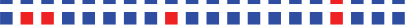

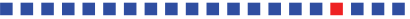

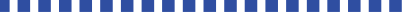

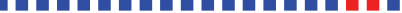

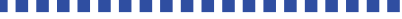

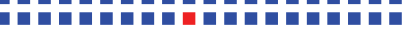

(c)

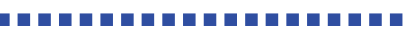

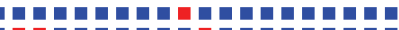

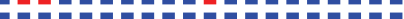

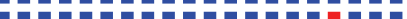

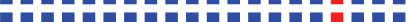

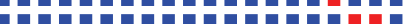

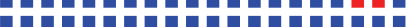

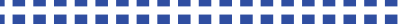

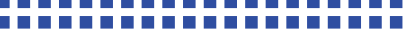

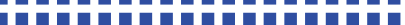

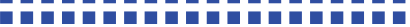

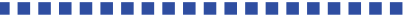

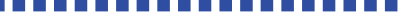

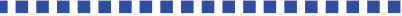

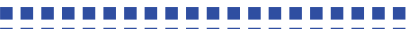

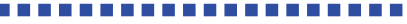

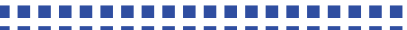

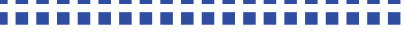

(f)

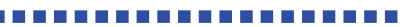

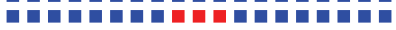

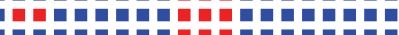

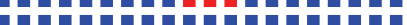

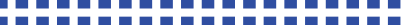

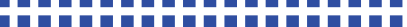

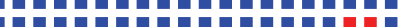
-

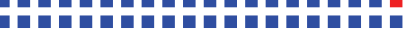

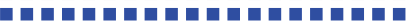

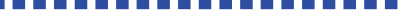

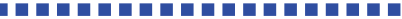

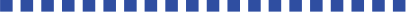

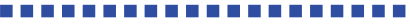

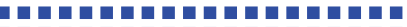

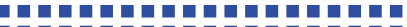

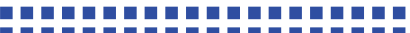

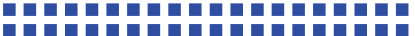

(i) 
the vanishing of a $1 D \rightarrow 9 D \rightarrow 1 D$ oscillator of defectors. In Figures $8(\mathrm{~g})-8(\mathrm{i})$, a $1 D \rightarrow 9 D \rightarrow 1 D$ oscillator of defectors is shown at the upper center. In Figure 8(j), affected by the randomness, the square shaped $D$-cluster turns to a line shaped $D$-cluster with two defectors instead of a single defector, which terminates the $1 D \rightarrow 9 D \rightarrow 1 D$ oscillation of defectors. After that, the line shaped $D$-cluster remains stable for several time generations until it turns to an arrow shaped $D$-cluster and vanishes in the next time generation as shown in Figures $8(\mathrm{k})-8(\mathrm{l})$.

Now we are ready to explain the Matthew effect of the random drift on the evolutionary PDG. Despite the population of its opponent, some particular shaped C-clusters and $D$-clusters can survive and maintain under the deterministic imitative mechanism. However, these equilibrium states strictly depend on the shapes of these clusters and thus are vulnerable to disturbance. The randomness brought by the employed random drift update rule can change the shapes of these clusters, leading to the breakdown of these clusters and the phenomenon of the Matthew effect.

\section{Conclusions}

Evolutionary dynamics are affected by population structure and update rules. Spatial or network structure facilitates the clustering of strategies, which represents a mechanism for the evolution of cooperation. However, whether the evolutionary dynamics is robust to disturbance deserves further studies. In this paper, the problem is explored by combining the deterministic imitative rule with the random drift reproduction rule in the prisoner's dilemma game on regular lattices. It is found that the employed random drift has an effect on the evolutionary dynamics depending on the values of the temptation-to-defect $b$ and the probability $p$. We are more interested in the cases where the random drift occurs only with low probabilities. Then, the random drift has an apparent Matthew effect on the evolution of cooperation: when the temptation for players to defect is relatively small, with cooperators prevailing in the original PDG, the applied random drift tends to further enhance the frequency of cooperators; under the opposite conditions which is cooperation unfavourable, the random drift tends to further depress cooperation. Analysis on the dynamics and pattern formation of the PDG system implies that the advantage brought by the clustering of strategies is diluted by the random drift, leading to the phenomenon of the Matthew effect.

\section{Conflict of Interests}

The authors declare that there is no conflict of interests regarding the publication of this paper.

\section{Acknowledgments}

The authors would like to thank Dr. Yu-Zhong Chen and Professor Ying-Cheng Lai for helpful discussions. This work was supported by the National Science Foundation of China under Grant no. 11101256 and Research and Innovation
Project of Shanghai Municipal Education Commission (no. 14YZ149).

\section{References}

[1] J. M. Smith and G. R. Price, "The logic of animal conflict," Nature, vol. 246, no. 5427, pp. 15-18, 1973.

[2] J. M. Smith, Evolution and the Theory of Games, Cambridge University Press, Cambridge, UK, 1982.

[3] F. C. Santos and J. M. Pacheco, "Scale-free networks provide a unifying framework for the emergence of cooperation," Physical Review Letters, vol. 95, no. 9, Article ID 098104, 2005.

[4] R. Boyd, H. Gintis, and S. Bowles, "Coordinated punishment of defectors sustains cooperation and can proliferate when rare," Science, vol. 328, no. 5978, pp. 617-620, 2010.

[5] Y. Z. Chen and Y. C. Lai, "Optimizing cooperation on complex networks in the presence of failure," Physical Review E, vol. 86, Article ID 045101, 5 pages, 2012.

[6] S. Coakley, M. A. Nowak, J. Almenberg et al., Evolution, Games, and God: the Principle of Cooperation, Harvard University Press, 2013.

[7] R. Axelrod, The Evolution of Cooperation, Basic Books, New York, NY, USA, 1984.

[8] H. Gintis, Game Theory Evolving, Princeton University, Princeton, NJ, USA, 2000.

[9] S. Meloni, A. Buscarino, L. Fortuna et al., "Effects of mobility in a population of prisoner's dilemma players," Physical Review E, vol. 79, no. 6, Article ID 067101, 2009.

[10] M. Perc and Z. Wang, "Heterogeneous aspirations promote cooperation in the prisoner's dilemma game," PLoS ONE, vol. 5, no. 12, Article ID e15117, 2010.

[11] J. M. Smith and E. Szathmáry, The Major Transitions in Evolution, Oxford University Press, Oxford, UK, 1995.

[12] E. Pennisi, "How did cooperative behavior evolve," Science, vol. 309, no. 5731, p. 93, 2005.

[13] M. A. Nowak, "Five rules for the evolution of cooperation," Science, vol. 314, no. 5805, pp. 1560-1563, 2006.

[14] M. Perc and A. Szolnoki, "Coevolutionary games-A mini review," BioSystems, vol. 99, no. 2, pp. 109-125, 2010.

[15] M. A. Nowak and R. M. May, "Evolutionary games and spatial chaos," Nature, vol. 359, no. 6398, pp. 826-829, 1992.

[16] M. A. Nowak and R. M. May, "The spatial dilemmas of evolution," International Journal of Bifurcation and Chaos, vol. 3, no. 1, pp. 35-78, 1993.

[17] P. D. Taylor, T. Day, and G. Wild, "Evolution of cooperation in a finite homogeneous graph," Nature, vol. 447, no. 7143, pp. 469472, 2007.

[18] G. Szabó and G. Fáth, "Evolutionary games on graphs," Physics Reports, vol. 446, no. 4-6, pp. 97-216, 2007.

[19] C. P. Roca, J. A. Cuesta, and A. Sánchez, "Effect of spatial structure on the evolution of cooperation," Physical Review E, vol. 80, no. 4, Article ID 046106, 2009.

[20] C. P. Roca, J. A. Cuesta, and A. Sánchez, "Evolutionary game theory: temporal and spatial effects beyond replicator dynamics," Physics of Life Reviews, vol. 6, no. 4, pp. 208-249, 2009.

[21] B. Allen, A. Traulsen, C. E. Tarnita, and M. A. Nowak, "How mutation affects evolutionary games on graphs," Journal of Theoretical Biology, vol. 299, pp. 97-105, 2012. 


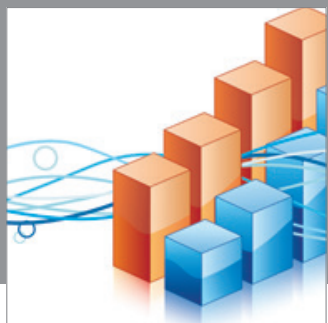

Advances in

Operations Research

mansans

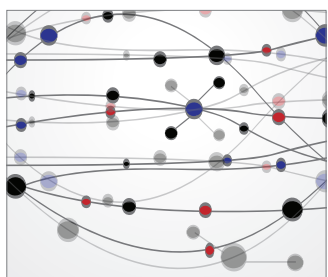

The Scientific World Journal
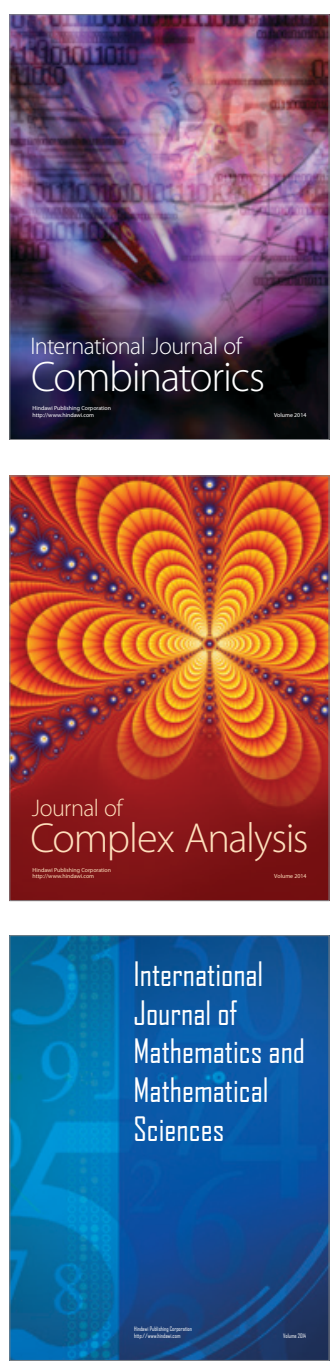
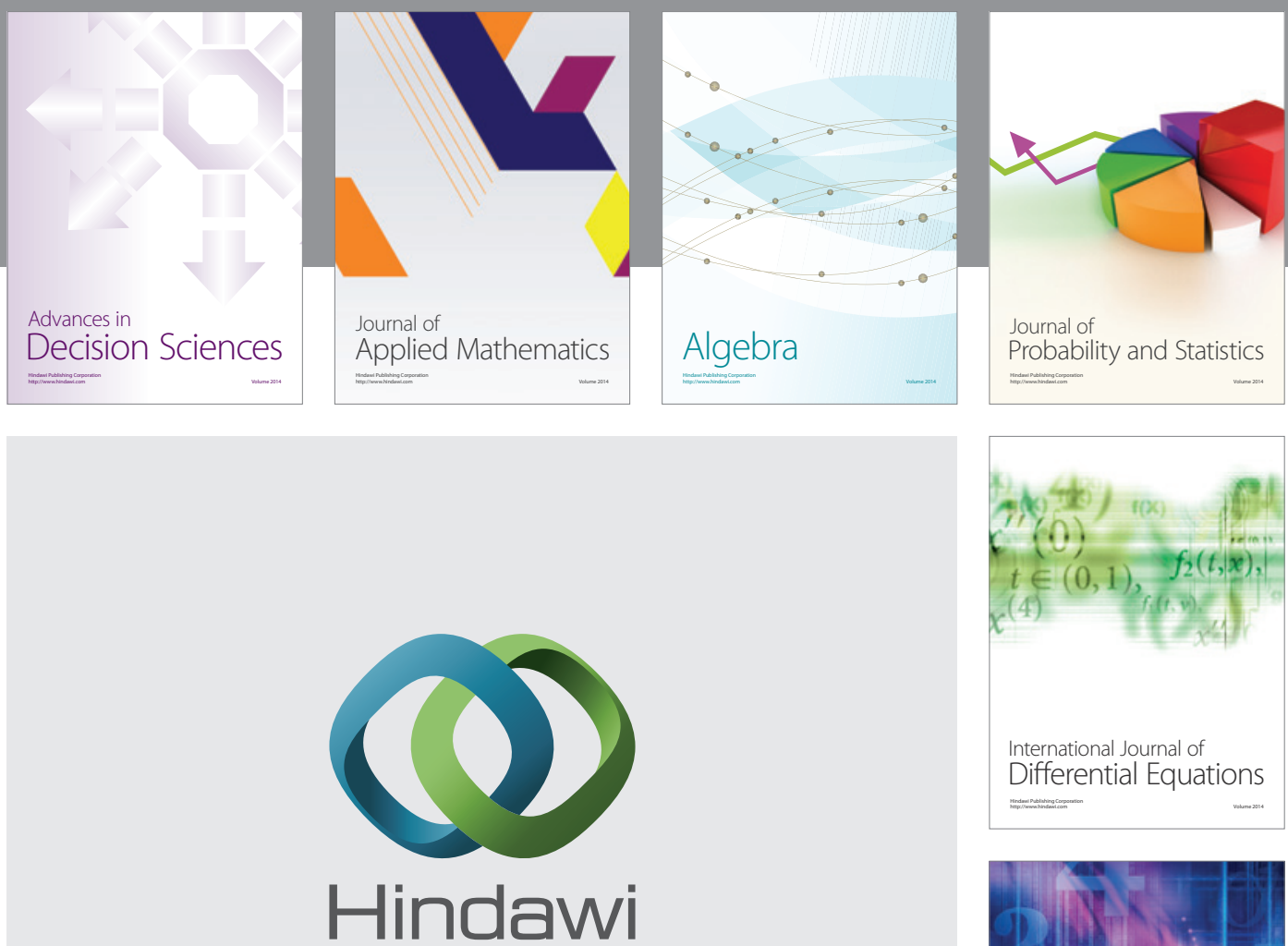

Submit your manuscripts at http://www.hindawi.com
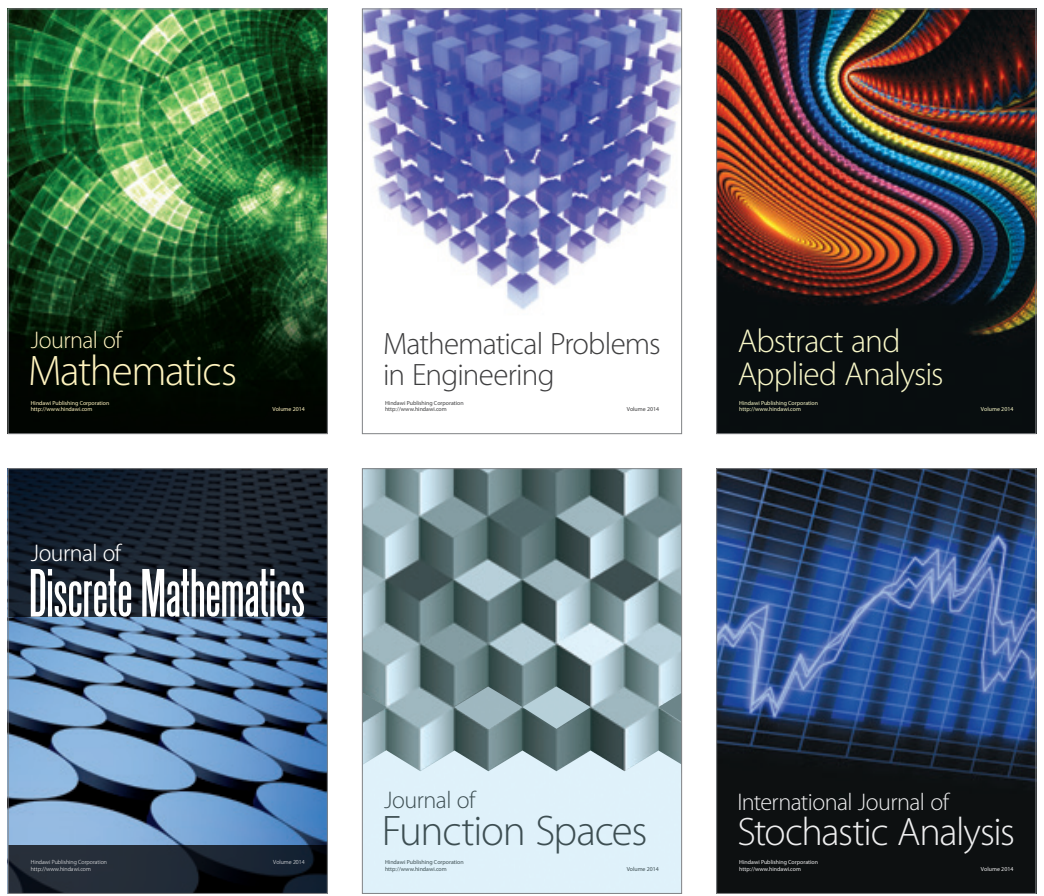

Journal of

Function Spaces

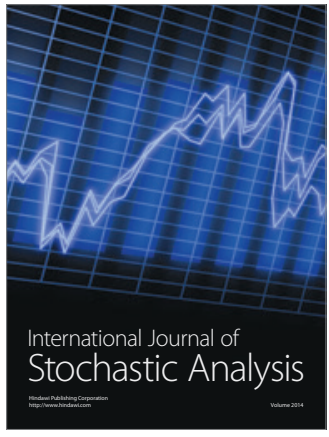

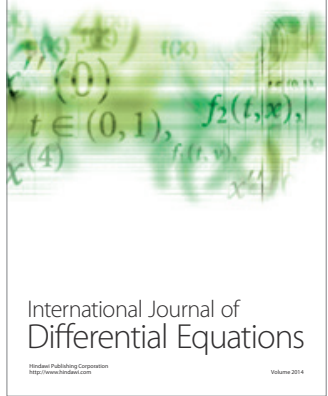
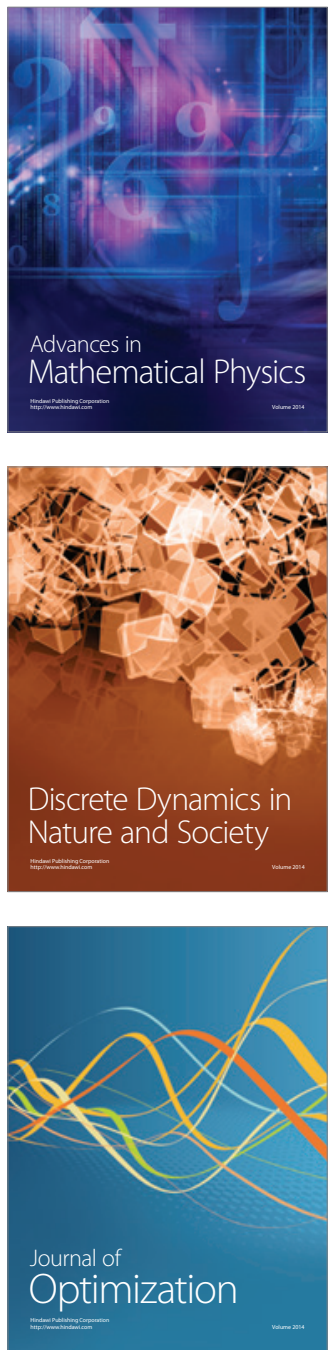